\title{
ECG based Atrial Fibrillation Detection using Cuckoo Search Algorithm
}

\author{
Padmavathi Kora, $\mathrm{PhD}$ \\ Gokaraju Rangaraju \\ Institute of Engineering \\ and Technology, Hyderabad
}

\author{
V. Ayyem Pillai, PhD \\ Gokaraju Rangaraju \\ Institute of Engineering \\ and Technology, Hyderabad
}

\begin{abstract}
Electrocardiogram (ECG), a non-invasive diagnostic technique, is used for detecting cardiac arrhythmia. For the last decade industry is dealing with Bio- medical instrumentation and research, demanding an advancement in its ability to distinguish different cardiac arrhythmia. Atrial Fibrillation (AF) is an irregular rhythm of the human heart. During AF, the atrial movements are quicker than the normal rate. As blood is not completely ejected out of atria, chances for the formation of blood clots in atrium. These abnormalities in the heart can be identified by the changes in the morphology of the ECG. The first step in the detection of AF is preprocessing of ECG, which removes noise using filters. Recent feature extraction methods, such as Auto Regressive (AR) modeling, Magnitude Squared Coherence (MSC) and Wavelet Coherence (WTC) using standard database (MIT-BIH), yielded a lot of features. Many of these features might be insignificant containing some redundant and nondiscriminatory features that introduce computational burden and loss of performance. So Cuckoo Search Algorithm (CSA) is directly used to optimize the raw ECG instead of extracting features using the above feature extraction techniques. This paper proposes the design of an efficient system for classification cardiac arrhythmia such as ANN (Artificial Neural Network), KNN (K- Nearest Neighbor), SVM (State Vector Machine). Our simulation results show that CSA with ANN gives $99.3 \%$ accuracy on MIT-BIH database by including NSR database also.
\end{abstract}

\section{Keywords}

Atrial Fibrillation, ECG, Cuckoo search, Neural Network Classifier.

\section{INTRODUCTION}

ECG describes the electrical action of the human heart. The changes in voltages

during repolarization and depolarization of the heart fibers are monitored by placing electrodes at different places on the chest and on the limb leads. The ECG signal is either printed on graph paper or displayed on a computer monitor. The benefits of ECG are its portability, prompt accessibility and flexibility. Computerized ECG classification can also help reduce health care costs. In the biomedical industry, there is an ongoing quest for the early detection of heart abnormalities using ECG signals.

This paper aims at developing an intelligent, inexpensive and flexible ECG- based automatic arrhythmia (irregular heart beat) detection system. The method includes signal preprocessing techniques to obtain the discriminative features of the ECG signals that correspond to a cardiac condition. These ECG features are classified using supervised learning techniques. Abnormal cardiac beat recognition is a crucial step in the detection of heart diseases. During AF the electrical discharges in the atrium are fast or rapid. Morphology changes can be observed through the changes in the ECG recordings. The clear indication of AF is irregular 'RR' interval and the absence of 'P' wave.

Since AF beats are occasional (rarely occurring), doctors sometimes have to depend on incidental examinations of AF. It would be quite inconvenient for doctors to go through the Electro-cardiogram manually, especially for the cases where the occurrence of AF beats are random, and a long-term monitoring is required to detect the abnormal activity for further processing.

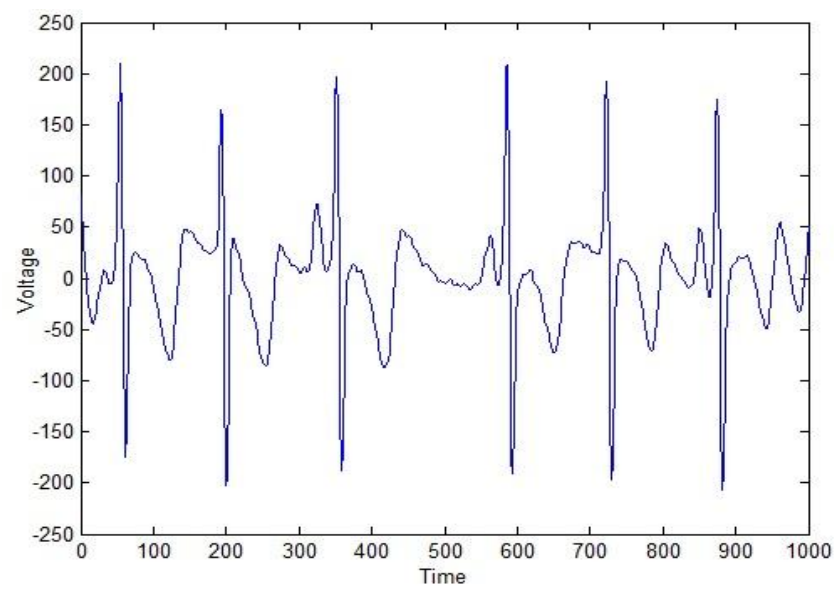

Figure 1: AF signal 
Fig 2 describes information about the relative amplitudes and time intervals of each ECG beat called morphological transitions. The morphology described by, $\mathrm{P}$, QRS complex, $\mathrm{T}$ and $\mathrm{U}$ waves, is called as features of ECG signal. Due to abnormalities in the heart morphology changes. $\mathrm{AF}$ is a morphological abnormality present in heart diseases. In this work, CSA is utilised as feature extraction method instead of traditional methods (1), (2). Waveform changes in the AF signal are given in Figure 1.

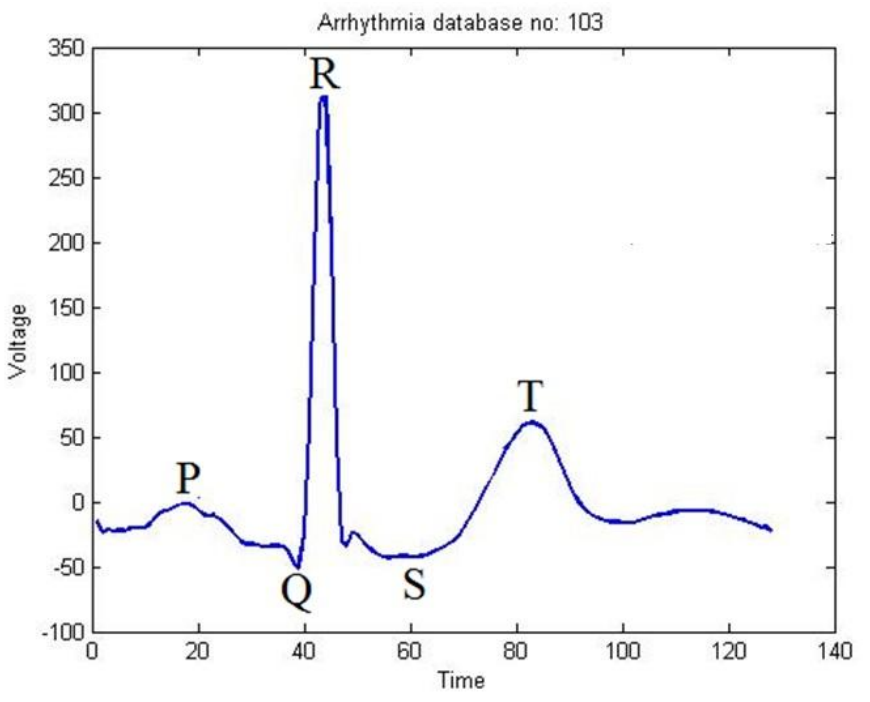

Figure 2: Normal ECG signal

Techniques based on RR interval shown in figure 1 proposed by Moody (3) and Tateno (4), and $\mathrm{P}$ wave-based methods presented by Mohebbi (5) have some limitations. When the ECG changes quickly between rhythms or when AF takes place with regular ventricular rates, the methods based on RR interval fails in detecting accurately. The absence of 'p' wave due to its small amplitude will make accurate detection difficult.

Different techniques and transformations proposed earlier in literature for extracting features from an ECG signal are explained in (6). Various algo- rithms for the feature extraction of ECG signal are Karhunen-Loeve trans- form (KLT) (7), Wavelet Transform (WT) (8) and the Discrete Cosine Trans- form (DCT) (9), (10) Fast Fourier Transform (FFT) (11) and . The DCT is widely used for its optimum energy compaction property because the division of the average energy of the signal is grouped into a comparatively few constituents of the DCT-coefficients. AF classification flow diagram is shown in Fig. 3.
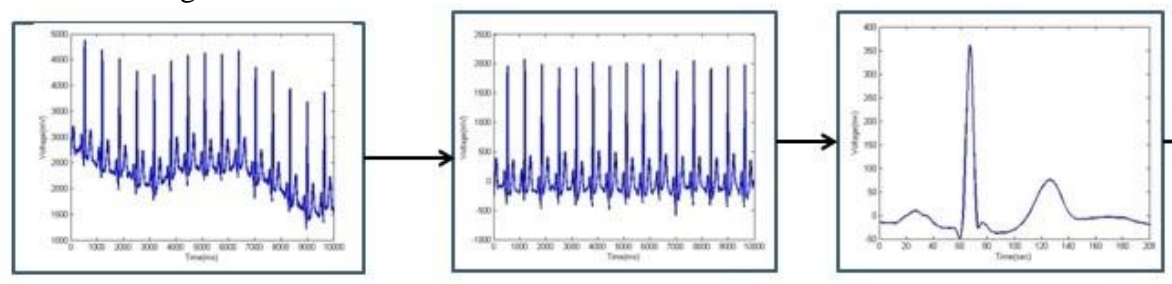

RawECG

Filtered ECG

ECG Beat (features)

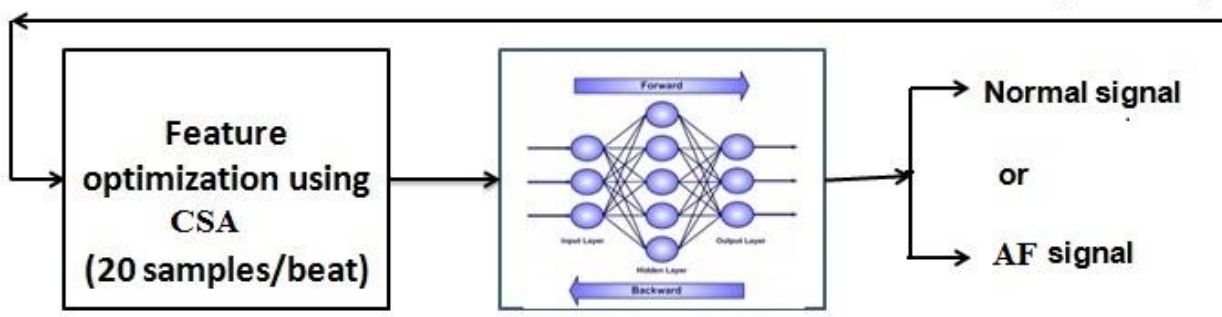

Optimization of features

\section{Classification} using LMNN

Figure 3: ECG classification using CSA features

\section{PRE-PROCESSING}

The preprocessing step is divided into three stages : Removing noise, Segmenting the ECG into beats (13) and Resampling all beats into equal size of 128 samples.
The noise from the ECG signal is removed using smoothing filter (also called digital smoothing polynomial filter or least-squares smoothing filter) Savizky - Golay (14) in which the signal-to-noise ratio is improved by 
using a method of Linear Least Squares (LLS). This filter is applied with a window size of 15 and a polynomial order of 13. This filter can be implemented with LLS. Noise is minimized by considering the sum of squared differences between the signal constellation. Then obtained signals are segmented into beats by detecting the ' $R$ ' peaks. The detected $R$ peaks of the ECG signal are shown in Fig. 4. From the MIT- BIH database (15) AF data files of 26 patients and Normal Sinus Rhythm files of 18 patients are used to detect AF. All the MIT$\mathrm{BIH}$ database was not sampled at 360 samples per second. The sampling rate of a normal signal is $128 \mathrm{~Hz}$ and AF signal frequency is $250 \mathrm{~Hz}$. After segmentation, ECG beats are of different lengths (sizes) are obtained.

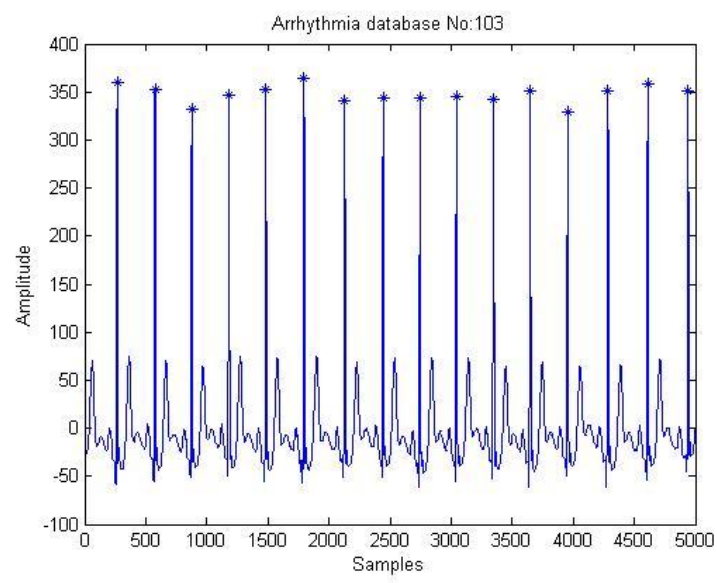

Figure 4: ECG $R$ peak detection

\section{FEATURE OPTIMIZATION}

Feature selection methods are used to determine most relevant features which can be used to classify the given datasets and to improve the accuracy of the classification results. The present study gives a new technique for the detection of two cardiac conditions (Normal and AF) using nature inspired optimization techniques in combination with three classifiers (Knearest neighbor (KNN), SVM (Support Vector Machines) and LMNN (Levenberg Marquardt Neural Net- work). CSA is a nature-inspired meta-heuristic optimization algo- rithm, which can be effectively used to identify the changes in the ECG by finding the optimized features (key features) of each cardiac beat. For the detection AF, these features are given to the KNN, SVM and LMNN classifiers.

\subsection{Cuckoo Search Algorithm(CSA)}

Another metaheuristic search algorithm, called Cuckoo Search Algorithm (CSA), was created by Yang and Deb in 2009. The algorithm is inspired by the reproduction system of cuckoos. This technique is easy, proficient and ideal for finding arbitrary search paths, and effectively applied to all engineering issues. There are numerous techniques to enhance its execution; few people concentrate the parameters of cuckoo search algorithm. Since these techniques are utilizing binary and decimal to encode the bird's nest, individual's data limit is exceptionally restricted.

At the fundamental level, cuckoos lay their eggs in the homes of other host birds, which might be of various species. If host bird may find that the eggs are not its own and either wreck the egg or relinquish the home all together. This has brought about the advancement of cuckoo eggs which mimic the eggs of host birds. For straightforwardness in depicting the Cuckoo Search, we now utilize the accompanying three rules:

1) Each cuckoo lays one egg at a time, and dumps it in a randomly chosen set;

2) the best nests with high-quality eggs will be carried over to the next generations;

3) the number of available host nests is fixed, and the egg laid by a cuckoo is discovered

by the host bird with a probability $\mathrm{p}$ lying between 0 and 1 ;

In this case, the host bird can either get rid of the egg away or simply abandon the nest and build a complex new nest. Based on the above rules, the basic CSA is 


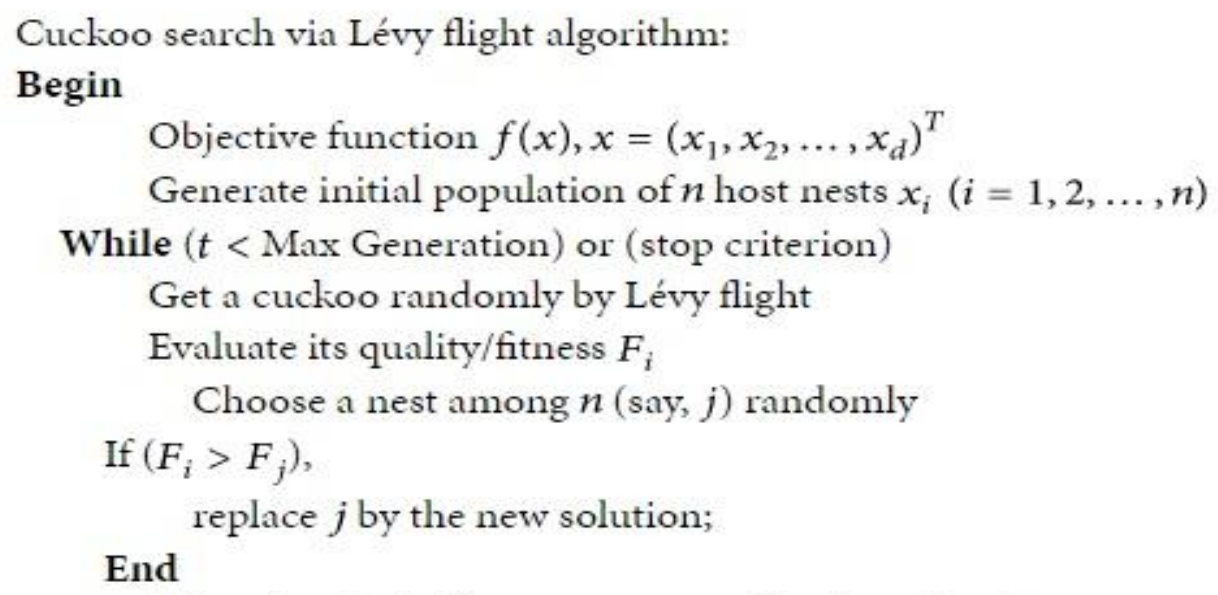

A fraction $\left(p_{a}\right)$ of worse nests are abandoned and new ones are built; Keep the best solutions (or nests with quality solutions);

Rank the solutions and find the current best

\section{End while}

\section{End}

Post process results and visualization

\section{RESULTS AND DISCUSSION}

The algorithm has been implemented using Matlab 7.12.0. The experiment has been done with a population size of 2086 for 50 generations. The optimized features from the Cuckoo search algorithm are given as the input for the LMNN so that its convergence speed and final accuracy can be increased. Compared to morphological feature extraction techniques, the CSA gives best features for detection of AF. The performance of CSA is compared with classical GA and PSO techniques. For measuring accuracy two parameters, Sensitivity (Sen) and Specificity (Spe) are calculated.

Table 1: Classification with GA features

\begin{tabular}{|c|c|c|c|}
\hline Classifier & Sen & Spe & Accuracy \\
\hline GA+KNN & $53.2 \%$ & $65.1 \%$ & $51.2 \%$ \\
\hline GA+SVM & $73.5 \%$ & $72.2 \%$ & $73.22 \%$ \\
\hline GA+LMNN & $92.35 \%$ & $93.9 \%$ & $92.1 \%$ \\
\hline
\end{tabular}

Table 2: Classification with PSO features

\begin{tabular}{|c|c|c|c|}
\hline Classifier & Sen & Spe & Accuracy \\
\hline PSO+KNN & $71.0 \%$ & $73.13 \%$ & $70.12 \%$ \\
\hline PSO+SVM & $76.2 \%$ & $75.4 \%$ & $72.13 \%$ \\
\hline PSO+LMNN & $95.5 \%$ & $96.9 \%$ & $96.74 \%$ \\
\hline
\end{tabular}

Table 3: Classification with CSA features

\begin{tabular}{|c|c|c|c|}
\hline Classifier & Sen & Spe & Accuracy \\
\hline CSA+KNN & $91.2 \%$ & $89.2 \%$ & $80.9 \%$ \\
\hline CSA+SVM & $89.34 \%$ & $89.2 \%$ & $89.2 \%$ \\
\hline CSA+LMNN & $99.97 \%$ & $98.7 \%$ & $99.3 \%$ \\
\hline
\end{tabular}


Tables 1, 2 and 3 compare the accuracy performance of KNN, SVM and LMNN classifiers with Genetic Algorithm (GA) and Particle Swarm Optimization (PSO). The classification accuracy of KNN classifier with CSA optimized features is $80.9 \%$ for the detection of AF. The classification accuracy of SVM classifier with CSA optimized features is $89.2 \%$ for the detection of AF. Results show that optimized CSA features in combination with LMNN classifier shows better results than KNN and SVM classifiers for the detec- tion of AF. The classification accuracy of LMNN classifier with CSA features is $99.3 \%$ for the detection of AF.

Table 4: Comparative study for the detection of AF

\begin{tabular}{|c|c|c|c|c|}
\hline Studies & Approach & Sen (\%) & Spe (\%) & Accuracy (\%) \\
\hline Tateno et. al(2001) (4) & RR Interval & 91.20 & 96.08 & -- \\
\hline $\begin{array}{c}\text { Paravaresh et. al } \\
(2011)(15)\end{array}$ & AR Coefficients & 96.12 & 93.20 & -- \\
\hline $\begin{array}{c}\text { Lee et. al (2013) (16) } \\
\begin{array}{c}\text { Zhou et. al (2014) } \\
(17)\end{array}\end{array}$ & RR Interval & 97.26 & 95.91 & 98.03 \\
\hline $\begin{array}{c}\text { Padmavathi Kora et. } \\
\text { al (2016) (18) }\end{array}$ & WTC Features & 96.89 & 96.27 & 99.1 \\
\hline \begin{tabular}{c} 
Proposed Approach \\
\hline
\end{tabular} & CSA & 100 & 99.7 & 99.8 \\
\hline
\end{tabular}

The work in (4) explored an experimental study based on the difference between RR intervals for extracting relevant features for the detection of AF. The values of sensitivity and specificity are $94.4 \%$ and $97.2 \%$ respectively.

The work presented in (15), used AR coefficients as features for classification $\mathrm{AF}$ using three different classifiers. AR coefficients are calculated for each 15 second data sequence length. The values of specificity and sensitivity are $96.14 \%$ and $93.20 \%$, respectively. The work proposed in (16) used three statistical methods for the detection of AF. These techniques are tested on AF database and Normal database. The values of sensitivity and specificity are $97.2 \%$ and $95.91 \%$ respectively. The work proposed in (17) used RR intervals, and computed various operations like nonlinear or linear integer filtering, symbolic dynamics and the calculation of Shannon entropy. On- line analytical processing of the method can be achieved using this novel algorithm. The values of sensitivity, specificity and accuracy are $96.89 \%, 98.27 \%$ and $98.03 \%$ respectively.

The work proposed in (18) used WTC coefficients for the detection of AF. The WTC features for the normal and AF datasets are calculated. These features are optimized using PCA algorithm. The values of sensitivity, specificity and accuracy are 100\%, $96.9 \%$ and $99.1 \%$ respectively.

From the simulation results, this study concludes that the proposed CSA optimization technique outperformed other three optimization algorithms with the selection of a minimal number of relevant features. The proposed method shows the highest classification accuracy for the detection of AF.

\section{CONCLUSION}

ECG is used to access the electrical activity of a human heart. In this study, our aim was to automate the above procedure so that it leads to cor- rect diagnosis. Early diagnosis and treatment is of great importance because immediate treatment can save the life of the patient. The proposed CSA method extracts the features from each ECG beat. These features were compared to GA and PSO optimization algorithms. The classification accuracy using CSA with LMNN classifier was $99.3 \%$ for the detection of AF. The simulation results have shown that the proposed CSA method can extract more relevant features than the other methods.

\section{REFERENCES}

[1] K. Padmavathi and K. Ramakrishna, "Classification of ecg signal during atrial fibrillation using burg's method," International Journal of Electrical and Computer Engineering, vol. 5, no. 1, p. 64, 2015.

[2] K. Padmavathi and K. Krishna, "Myocardial infarction detection using magnitude squared coherence and support vector machine," in Medical Imaging, m-Health and Emerging Communication Systems (MedCom), 2014 International Conference on. IEEE, 2014, pp. $382-385$.

[3] G. B. Moody and R. G. Mark, "A new method for detecting atrial fibrillation using rr intervals," Computers in Cardiology, vol. 10, no. 1, pp. 227-230, 1983.

[4] K. Tateno and L. Glass, "A method for detection of atrial fibrillation using $\mathrm{rr}$ intervals," in Computers in Cardiology 2000. IEEE, 2000, pp. 391-394.

[5] M. Mohebbi and H. Ghassemian, "Detection of atrial fibrillation episodes using svm," in Engineering in Medicine and Biology Society, 2008. EMBS 2008. 30th Annual International Conference of the IEEE. IEEE, 2008, pp. 177180.

[6] S. Karpagachelvi, M. Arthanari, and M. Sivakumar, "Ecg feature extraction techniques-a 
survey approach," arXiv preprint arXiv:1005.0957, 2010.

[7] M. Effros, H. Feng, and K. Zeger, "Suboptimality of the karhunen-loeve transform for trans- form coding," Information Theory, IEEE Transactions on, vol. 50, no. 8, pp. 1605-1619, 2004.

[8] R. J. Martis, U. R. Acharya, and L. C. Min, "Ecg beat classification using pca, lda, ica and discrete wavelet transform," Biomedical Signal Processing and Control, vol. 8, no. 5, pp. 437-448, 2013.

[9] K. G. Beauchamp, Applications of Walsh and related functions. Academic press, 1984.H. Khorrami and M. Moavenian, "A comparative study of dwt, cwt and det transformations in ecg arrhythmias classification," Expert systems with Applications, vol. 37, no. 8, pp. 5751-5757, 2010.

[10] R. Clayton and A. Murray, "Estimation of the ecg signal spectrum during ventricular fibrillation using the fast fourier transform and maximum entropy methods," in Computers in Cardiology 1993, Proceedings. IEEE, 1993, pp. 867-870.

[11] C. Saritha, V. Sukanya, and Y. N. Murthy, "Ecg signal analysis using wavelet transforms," Bulg. J. Phys, vol. 35, no. 1, pp. 68-77, 2008.

[12] H. Krim and D. H. Brooks, "Feature-based segmentation of ecg signals," in Time-Frequency and Time-Scale Analysis, 1996., Proceedings of the IEEE-SP International Symposium on. IEEE, 1996, pp. 97-100.
[13] S. Hargittai, "Savitzky-golay least-squares polynomial filters in ecg signal processing," in Computers in Cardiology, 2005. IEEE, 2005, pp. 763-766.

[14] A. L. Goldberger, L. A. Amaral, L. Glass, J. M. Hausdorff, P. C. Ivanov, R. G. Mark, J. E. Mietus, G. B. Moody, C.-K. Peng, and H. E. Stanley, "Physiobank, physiotoolkit, and physionet components of a new research resource for complex physiologic signals," Circulation, vol. 101, no. 23, pp. e215-e220, 2000.

[15] S. Parvaresh and A. Ayatollahi, "Automatic atrial fibrillation detection using autoregressive modeling," in 2011 International Conference on Biomedical Engineering and Technology, 2011, pp. 4-5.

[16] J. Lee, B. A. Reyes, D. D. McManus, O. Mathias, and K. H. Chon, "Atrial fibrillationdetection using an iphone 4s," Biomedical Engineering, IEEE Transactions on, vol. 60, no. 1, pp. 203-206, 2013.

[17] X. Zhou, H. Ding, B. Ung, E. PickwellMacPherson, Y. Zhang et al., "Automatic online detection of atrial fibrillation based on symbolic dynamics and shannon entropy," Biomed Eng Online, vol. 13, no. 18, pp. 1-18, 2014.

[18] K Padmavathi and K. S. Ramakrishna "Detection of atrial fibrillation using continuous wavelet transform and wavelet coherence," International Journal of Systems, Control and Communications, vol. 6, no. 4, pp. 292-304, 2015. 